\title{
Erratum to: Hydrodynamic dispersion in $\beta$-lactoglobulin gels measured by PGSE NMR
}

\author{
E.O. Fridjonsson ${ }^{1}$, D. Bernin ${ }^{2}$, J.D. Seymour ${ }^{1, a}$, M. Nydén ${ }^{2}$, and S.L. Codd ${ }^{3}$ \\ 1 Department of Chemical and Biological Engineering, Montana State University, USA \\ 2 Department of Chemical and Biological Engineering, Chalmers University of Technology, Sweden \\ 3 Department of Mechanical and Industrial Engineering, Montana State University, USA
}

Original article: Eur. Phys. J. E (2011) 34: 18, DOI: 10.1140/epje/i2011-11018-7.

Due to a technical oversight a series of author corrections have not been implemented in the final version of the paper. We publish here the full text of the article in its final corrected version sincerely apologizing to the authors for the inconvenience.

Received 23 July 2010

Published online: 24 March 2011 - C EDP Sciences / Società Italiana di Fisica / Springer-Verlag 2011

\begin{abstract}
The displacement scale dependent molecular dynamics of solvent water molecules flowing through $\beta$-lactoglobulin gels are measured by pulse gradient spin echo (PGSE) nuclear magnetic resonance (NMR). Gels formed under different $p \mathrm{H}$ conditions generate structures which are characterized by magnetic resonance imaging (MRI) and PGSE NMR measured dynamics as homogeneous and heterogeneous. The data presented clearly demonstrate the applicability of the theoretical framework for modeling hydrodynamic dispersion to the analysis of protein gels.
\end{abstract}

\section{Introduction}

Gels are found in a wide variety of biological, chemical and food systems and have received much attention [1-7]. Gels can generally be categorized as either well-ordered lamellar structures, covalent polymeric networks, polymer networks formed through physical aggregation or particulate structures [8]. These different categories are a symptom of the diversity of gel architecture which not only depends on the constituent macromolecules but also the gelation process, where variables such as $p \mathrm{H}$, ionic strength, temperature, solvent and copolymers can cause drastically different morphologies. Previous studies [9-16] have focused on understanding the fundamental correlation between the structure of gels and the dynamic properties of the constituent macromolecules and solvent. Gels generally consist of macromolecular networks with a large amount of solvent causing a large average mesh size [17] $(\xi)$ of the network compared with the size $(R)$ of solvent molecules (or tracers), allowing for a relatively un-restricted diffusive motion of these molecules $[9,10,18]$. The ratio $R / \xi$ is an important characteristic length for the gel structure, characterizing the subdivision of flow into different regimes, i.e. $R / \xi>1$, where for polymer gels self-diffusion is believed to proceed by the reptation mechanism [19], and $R / \xi<1$, where macroscopic flow is realized. The fluid dynamics through gels is particularly relevant to the re-

\footnotetext{
a e-mail: jseymour@coe.montana.edu
}

cent use of stimuli-responsive hydrogels as active functional components in fluidic and micro-fluidic flow devices [20-22]. In such situations the degree of solvent uptake and flow resistance depends on the chemistry of the macromolecules, solvent, temperature and $p \mathrm{H}$. This study focuses on gels prepared from bovine $\beta$-lactoglobulin, a member of the lipocalin family. $\beta$-lactoglobulin is one of the most highly studied gel-forming macromolecules, having been characterized with a wide range of techniques, both biochemical and physical [23]. The interest in studying this protein is due to its importance to the milk industry, where milk proteins play a range of roles in the manufacture of dairy products and products containing dairy components. These roles include nutrition, physical functionality, and breakdown under controlled conditions to produce nutritional or functional products. Understanding these proteins would help control the fouling of heated surfaces, gelling and clogging in processing equipment, and even undesired non-gelling during the manufacture of certain products [24].

$\beta$-lactoglobulin is a globular protein which is the main component of whey. When heat-denatured it forms selfsimilar aggregates characterized by a fractal dimension and whose degree of branching determines the eventual morphology of the gel [25]. Several genetic variants exist of which $\mathrm{A}$ and $\mathrm{B}$ are the most abundant and have been shown to aggregate at the same rate [26]. Generally, heat denaturation of globular proteins causes interactions 
between proteins such as the formation of covalent disulfide bridges [27]. In the absence of salts, globular proteins often form transparent gels of cross-linked strands (diameter $<10 \mathrm{~nm}$ ) at a $p \mathrm{H}$ higher or lower than $\mathrm{pI}$, the isoelectric point, while forming opaque gels consisting of agglomerated spherical protein particles close to pI [28, 29 . $\beta$-lactoglobulin $(\mathrm{pI}=5.2)$ adheres to this general picture, forming white particulate gels at $p \mathrm{H}=4-6$, while forming transparent fine-stranded gels above and below this $p \mathrm{H}$ range [30]. It has been shown that close to $\mathrm{pI}$, $\beta$-lactoglobulin precipitates out of solution even at room temperature and cannot form stable aggregates reproducibly, while for $p \mathrm{H}>5.8$ the aggregation process does not depend strongly on $p \mathrm{H}[28]$. In this study the two gels used are at $p \mathrm{H}=5.2$ and $p \mathrm{H}=7.0$, and, respectively, have heterogeneous and homogeneous structural morphologies at the macroscopic scale $(>100 \mu \mathrm{m})$. Therefore theoretical interest for this study is in the aggregation process consistent with $p \mathrm{H} \geq \mathrm{pI}$. A different process has been shown to govern aggregation at $p \mathrm{H}<\mathrm{pI}$. Two types of aggregation may occur for $\beta$-lactoglobulin. Spontaneous aggregation of native proteins and aggregation due to heat-induced denaturation, with different types of bonds formed in each case. For heat-induced denaturation the important concentrations are the critical association concentration (CAC) below which aggregation does not occur, gelation concentration $\left(C_{g}\right)$ above which gel formation occurs and salt concentration $\left(C_{s}\right)$. $C_{s}$ can dramatically change the structure of a gel, causing densification of aggregates due to increased branching, resulting in a decrease in CAC and decreased flexibility $[25,31]$. The gels used here have concentration $C=90 \mathrm{~g} / \mathrm{L}$ which is well above the expected $\mathrm{CAC}$ and $C_{g}$ for $p \mathrm{H}=5.2$ and 7.0 [25,28], while $C_{s}=0.3 \mathrm{M} \mathrm{NaCl}$ falls into a high ionic strength regime. The aggregation of $\beta$-lactoglobulin is believed to follow two steps [28]. First a pre-aggregation occurs during which monomers aggregate into 50-100 monomer units forming strong bonds which are irreversible. If $C<C_{g}$ these preaggregates will not aggregate further [32]. However if $C>$ $C_{g}$ these pre-aggregates form larger aggregates, creating a gel of linear or branched units depending on the $p \mathrm{H}$ and salt concentration. The rate at which aggregates form is strongly dependent on temperature. The gels used in this study are formed at $p \mathrm{H}=5.2$ and 7.0 at high salt concentrations $(0.3 \mathrm{M} \mathrm{NaCl})$ and are therefore both categorized as white particulate gels [30] due to the induced branching.

The focus of this work is to quantify the hydrodynamic dispersion of solvent water during pressure-driven flow through $\beta$-lactoglobulin gels of homogeneous $(p \mathrm{H}=5.2)$ and heterogeneous $(p \mathrm{H}=7.0)$ structure. The application of pulsed gradient spin echo (PGSE) nuclear magnetic resonance (NMR) [33] to measure the displacement length and time scale dependent dynamics demonstrates the applicability of the theory of hydrodynamic dispersion in porous media to analyze flow in gels. This represents a significant extension over current analysis of advective transport in gels which have focused on measurements of hydrodynamic permeability assuming classic D'Arcy law velocity scaling with pressure drop $[13,16,34]$.

\section{Theory}

\subsection{Transport in gels}

There has been extensive use of NMR to study gels, specifically using restricted solvent or tracer transport $[35,36]$ and directly measuring the network structure $[12,35,36]$. These studies clearly indicate that the proton-containing gel networks have associated water molecules, which allow for the use of cross-relaxation spectroscopy to detect water-protein interactions and the immobilized component of the gel [12]. This study will not be concerned with the direct detection of the gel network motion, rather the diffusive and advective motion of the solvent molecules is directly measured and interaction with the macromolecules indirectly indicates the gel network structure.

In gels the diffusion of solvent or probe particles is obstructed/restricted by the network, i.e. molecules freely diffuse at short time scales $(t \ll \tau)$ but are restricted at long time scales $(t \gg \tau)$ by the network, the time scales being defined relative to the solvent structure interaction time $(\tau)$. The ratio of the solvent molecule size and the gel mesh size plays an important role in determining the diffusion of the solvent molecules inside the network as pointed out in the introduction. Measurements and theory indicate that the diffusion coefficient can be described by simple scaling laws which generally show the diffusion coefficient decreasing (become more restricted) as macromolecular concentration increases [14]. Due to the variability inherent in modeling diffusion in gel systems multiple models have emerged based on free volume theory, hydrodynamic theory and obstruction effects [14]. For example, Altenberger and Tirrell [9] have considered two mechanisms restricting the mobility of diffusible particles, i.e. the scattering by the obstacle and the "self-interaction" due to the reflection of hydrodynamic disturbances from the obstacle, when either mechanism is dominant the expected self-diffusion coefficients are, respectively,

$$
D^{*} \sim(1-\phi) D_{0}, \quad D^{*} \sim\left(1-\phi^{1 / 2}\right) D_{0},
$$

where $D_{0}$ is the diffusion coefficient of unrestricted solvent molecules and $\phi$ is the macromolecular concentration. While Cukier [10] used a renormalized Navier-Stokes equation to account for screening effects in the interaction between tracers and gel monomers, to obtain

$$
\frac{D_{0}}{D^{*}}=e^{\epsilon R}
$$

where $R$ is the radius of the tracers, $\epsilon$ is the screening parameter, which characterizes the resistance of the polymer network to the motion of the tracers. $\epsilon \sim \phi^{\kappa}$ where $\kappa=0.5$ (mobility is restricted by strong hydrodynamic effects) and $\epsilon=1.0$ (scattering effects are dominant). As indicated by eq. (2) if the tracer is a small molecule $D^{*} \sim D_{0}$, while eq. (1) indicates that, respectively, for $\phi=0.09, D^{*}=0.91 D_{0}$ and $0.70 D_{0}$.

Experimental results for diffusion in gels are usually obtained while varying $\phi$ or the degree of swelling. The 
study that is performed here keeps $\phi$ constant while varying the experimental displacement observation time $(\Delta)$. A similar relationship between $D^{*}$ and $\phi$ exists between $D^{*}$ and $\Delta$, i.e. $D^{*}$ decreases with increasing $\Delta$, with the difference being that as $\Delta \rightarrow \infty, D^{*}$ should asymptote once the solvent molecules have all experienced similar environments. This type of $D^{*}(\Delta)$ study using NMR is displacement scale dependent and requires significant accounting of surface effects between the gel network and the solvent molecules, as well as any potential relaxation weighting effects $\left(T_{1}, T_{2}\right)$ as $\Delta$ increases [37,38].

Advective transport of solvent through gel networks has been studied $[13,16,34]$, these studies show that the network infers hydrodynamic friction as well as deforming from its initial configuration by the drag force, with the flow process being determined by the balance between the viscoelastic response of the gel network and the solvent flow. In investigating convective transport through gels this viscoelastic network response adds complexity relative to studies of rigid or purely elastic porous media.

Despite the significance, the solvent flow process inside gels has yet to be studied in detail [16]. In this study we demonstrate the ability of NMR to characterize the system dynamics non-destructively. The data provide transport information inside the gel in a Lagrangian reference frame, yielding new information for furthering understanding of solvent transport inside gels.

\subsection{Hydrodynamic dispersion in porous media}

The non-steady, irreversible spreading of flowing fluid molecules within a porous medium is known as hydrodynamic dispersion. It is the process in which a mass of solute at time $t=0$, within the flow domain, spreads and occupies an ever-increasing volume of the porous medium as time increases. It is governed by both mechanical dispersion due to mechanical mixing of advective flow streamlines, the slow motion of molecules near solid surfaces [39] and molecular diffusion across streamlines [40]. A thorough review of the subject matter can be found elsewhere $[41,42]$ and only ideas related to interpretation of our data will be given here. The dispersion of a fluid in a porous media indicates the degree of interconnectivity of the pore space and provides an insight into the morphological characteristics of the porous medium. A large number of dispersion experiments in porous media have been conducted on Newtonian and non-Newtonian fluids [43]. The Péclet number $(P e)$ is the ratio of advective to diffusive forces that characterizes dispersion in porous media and can be written [44]

$$
P e=\frac{\langle v\rangle l}{D_{0}},
$$

where $\langle v\rangle$ is the fluid's mean velocity through the system, $l$ is the characteristic length of the system and $D_{0}$ is the self-diffusion coefficient of the fluid. The dispersion tensor components, $D_{\|}^{*}$ and $D_{\perp}^{*}$, where $\|$ and $\perp$ refer, respectively, to the longitudinal direction parallel and the transverse direction perpendicular to the flow directions of the dispersion tensor, are of interest. The effective dispersion coefficient, $D^{*}$, can be defined as [41]

$$
D^{*}(\Delta)=\frac{1}{2} \frac{\mathrm{d} \sigma^{2}(\Delta)}{\mathrm{d} \Delta},
$$

where $\sigma^{2}=\left\langle(\boldsymbol{R}-\langle R\rangle)^{2}\right\rangle$ is the variance of the distribution of the displacement, $\boldsymbol{R}$, for a particular time $\Delta$. As $\Delta \rightarrow \infty, D^{*}$ asymptotes to a constant value in systems with homogeneous structure exhibiting normal diffusion characterized by the Einstein relation $\sigma=2 D^{*} \Delta[45]$. Under these circumstances $\mathrm{d} \sigma^{2} / \mathrm{d} \Delta$ becomes a constant $\left(D^{*} \neq f(\boldsymbol{R}, \Delta)\right.$ ), and the dispersion is Fickian in nature. The probability distribution of displacement, i.e. the average propagator, is Gaussian [41]

$$
P(\boldsymbol{R}, \Delta)=\frac{1}{\sigma \sqrt{2 \pi}} \exp \left[-\frac{1}{2} \frac{(\boldsymbol{R}-\langle v\rangle \Delta)^{2}}{\sigma^{2}}\right] .
$$

The longitudinal transport in the system is modeled by the advection diffusion equation

$$
\frac{\partial P}{\partial t}+\langle v\rangle \frac{\partial P}{\partial z}=D^{*} \frac{\partial^{2} P}{\partial z^{2}}
$$

when the dynamics exhibit asymptotic normal diffusion.

In systems in which the heterogeneous structure generates correlated dynamics of the flowing fluid non-local (non-Fickian) dispersion dynamics occur [46]. Power law correlations in permeability variations [47] and other longrange structural correlations can generate anomalous diffusion dynamics resulting in $\sigma^{2}(\Delta)=2 D^{*}(\Delta) \Delta \propto \Delta^{\alpha}$, with $\alpha<1$ defined as subdiffusive and $\alpha>1$ defined as superdiffusive. The longitudinal transport in such systems is modeled by continuous time random walks (CTRW) and fractional advection diffusion equations [48-53].

For the work presented here the CTRW formalism provides a model for the solvent flow through heterogeneous gels where the solvent molecules are divided between a slow transport regime (long wait time) and a fast transport regime (shorter or non-existent wait time). The molecular motion is assumed independent of the preceding waiting time but dependent on the velocity at the starting point. Generally, as $\alpha$ decreases from the Galilei-invariant Gaussian at $\alpha=1$ to the Galilei-variant forms there is an increase in a long displacement tail which is in agreement with the effects on the propagators for the heterogeneous gels observed in this study and has been previously observed for heterogeneous porous media $[54,55]$.

Another useful method to analyze the propagator data in this study is to look at the stretched exponential wavelength scaling $E(\boldsymbol{q}, \Delta) \sim \exp \left(-c \boldsymbol{q}^{\beta}\right)$ which corresponds to a fractional ADE model with a Poisson wait time distribution and a Lévy distribution for the jump length [45]. This approach has been previously used in a phenomenological fashion to characterize the non-Gaussian nature of PGSE data $[54,55]$. Low- $\boldsymbol{q}$-space data can be fit with this scaling, where $\beta \sim 1$ corresponds to an exponential, while $\beta \sim 2$ corresponds to a Gaussian probability distribution. 


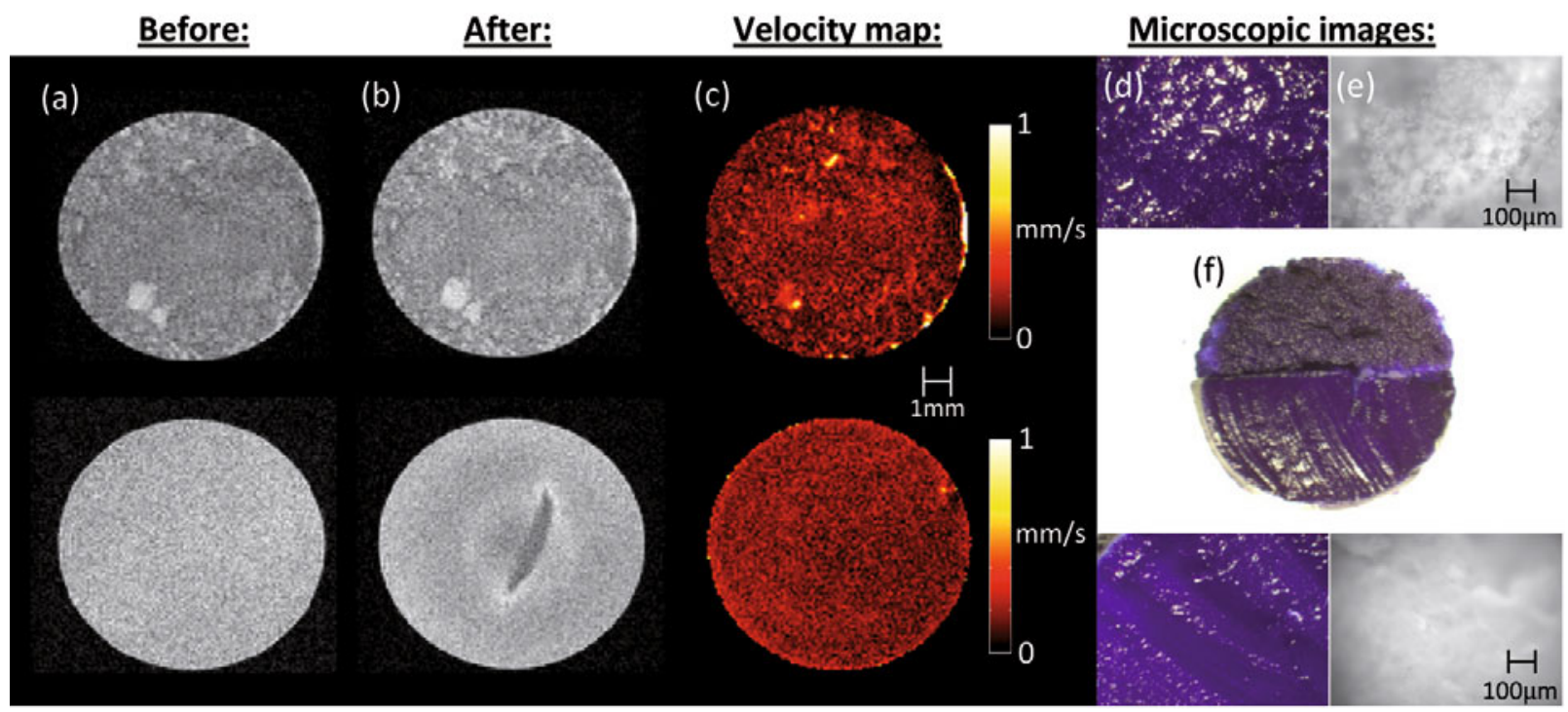

Fig. 1. NMR images (a, b), velocity map (c), stereoscopic microscopy images (d, f) and light microscopy images (e) of the heterogeneous (top) and homogeneous (bottom) gels. NMR experimental parameters: $256 \times 256$ pixels, $1 \mathrm{Average,} \mathrm{SW:} 50 \mathrm{kHz}$, $T_{R}: 1 \mathrm{~s}$ or $5 \mathrm{~s}, T_{E}: 12 \mathrm{~ms}, 1 \mathrm{~mm}$ slice, FOV: $12 \mathrm{~mm} \times 12 \mathrm{~mm}$ or $11 \mathrm{~mm} \times 11 \mathrm{~mm}$, Voxel Resolution: $47 \times 47 \times 1000 \mu \mathrm{m}^{3}$ or $43 \times 43 \times 1000 \mu \mathrm{m}^{3}$. Gradients for velocity maps: 60 and $460 \mathrm{mT} / \mathrm{m}, \delta: 1 \mathrm{~ms}, \Delta: 20 \mathrm{~ms}$.

\section{Materials and methods}

A $10 \mathrm{~mm}$ ID, $30 \mathrm{~mm}$ length liquid chromatography (LC) column (Omnifitß) with a frit at the entrance was filled with $9 \%$ wt $\beta$-lactoglobulin (Sigma-Aldrichß) and $0.3 \mathrm{M}$ $\mathrm{NaCl}$ dissolved in water overnight. The $p \mathrm{H}$ of the mixture was adjusted to either $p \mathrm{H}=5.2$ or 7.0 to, respectively, produce a heterogeneous or homogeneous gel. The mixture is then heated from room temperature to $90^{\circ} \mathrm{C}$ using a RTE111 water bath (NesLabß) to allow for the aggregation of the proteins into a gel structure inside the LC column. These gel-filled LC columns were then connected to a dualsyringe high-pressure LC pump (Pharmacia P-500®) and NMR experiments were performed. A Bruker $250 \mathrm{MHz}$ superconducting magnet with a Mirco2.5 (Bruker Biospin, Karlsruhe Germany) microimaging probe $\left(\boldsymbol{g}_{\max }: 0.86 \mathrm{~T} / \mathrm{m}\right.$ (40 A) in three orthogonal $(X, Y, Z)$ gradient directions) and $20 \mathrm{~mm}$ radiofrequency (rf) coil was used. Experiments at no flow conditions and at constant volume flow conditions were done with volumetric flow rates of 10, 20 and $30 \mathrm{~mL} / \mathrm{h}$. In the homogeneous gel pure water and $0.3 \mathrm{M}$ $\mathrm{NaCl}$ fluids were used to check the impact of the flowing fluid on the gel elastic response. To characterize the gel structure NMR images (MRIs), velocity maps, stereo and compound microscopy images were obtained (fig. 1). The homogeneous gel was found to compact with increasing flow rate and tear in the middle at a flow rate of $30 \mathrm{~mL} / \mathrm{h}$ (see fig. 2), while the heterogeneous gel maintained its structure at all flow rates tested (up to $100 \mathrm{~mL} / \mathrm{h}$ ). This morphological difference is associated with the higher pressures required to maintain the volumetric flow rates through the homogeneous gels, while the heterogeneous gel requires much lower pressure drops due to its open structure. The strain $(\Delta L / L)$ on the gels was quantified from the MRIs by the change in the height $(\Delta L)$ of the gel relative to their original heights $(L)$. The gel structure and flowing solvent transport molecular dynamics were investigated using PGSE NMR techniques to obtain the probability distribution of displacement, reciprocal $\boldsymbol{q}$-space data and dispersion coefficients in directions parallel and perpendicular to the flow direction. The PGSE pulse sequence (fig. 3a) and velocity imaging sequence, which combines PGSE and MRI sequences (fig. 3b) show the timing of the application of radiofrequency and magnetic field gradients. These experiments were conducted under no-flow condition as well as during the application of the different volumetric flow rates $(10,20$ and $30 \mathrm{~mL} / \mathrm{h})$.

\subsection{NMR experiments}

The NMR theory presented here serves as a brief overview for the NMR pulse sequences used to measure the transport phenomena in this study, more details can be found elsewhere [33]. The PGSE sequence (fig. 3a) applies a magnetic field gradient pulse $\boldsymbol{g}$ of duration $\delta$, which initially imparts a spatially dependent phase shift $\phi(\boldsymbol{r})$ on the spins (or NMR active water molecules) $\phi(\boldsymbol{r})=\gamma \delta \boldsymbol{g} \cdot \boldsymbol{r}$ After delay time $\Delta$, the spins, will have migrated to $\boldsymbol{r}^{\prime}$, and after a second gradient pulse is applied to the system the net phase shift for the spins is $\phi(\boldsymbol{r})=\gamma \delta \boldsymbol{g} \cdot\left(\boldsymbol{r}^{\prime}-\boldsymbol{r}\right)$ As negligible motion is assumed to occur during the gradient pulse duration $\delta$ and $\delta \ll \Delta$ the so-called "narrow-pulse approximation" applies [33] it follows that the normalized echo signal that is acquired after the PGSE sequence encoding has the form

$$
\begin{aligned}
E(\boldsymbol{g}, \delta, \Delta) & =\int \rho(\boldsymbol{r}) \int P\left(\boldsymbol{r} \mid \boldsymbol{r}^{\prime}, \Delta\right) \exp \left[i 2 \pi \boldsymbol{q} \cdot\left(\boldsymbol{r}^{\prime}-\boldsymbol{r}\right)\right] \mathrm{d} \boldsymbol{r}^{\prime} \mathrm{d} \boldsymbol{r} \\
& =\int \bar{P}(\boldsymbol{R}, \Delta) \exp [i 2 \pi \boldsymbol{q} \cdot \boldsymbol{R}] \mathrm{d} \boldsymbol{R}
\end{aligned}
$$




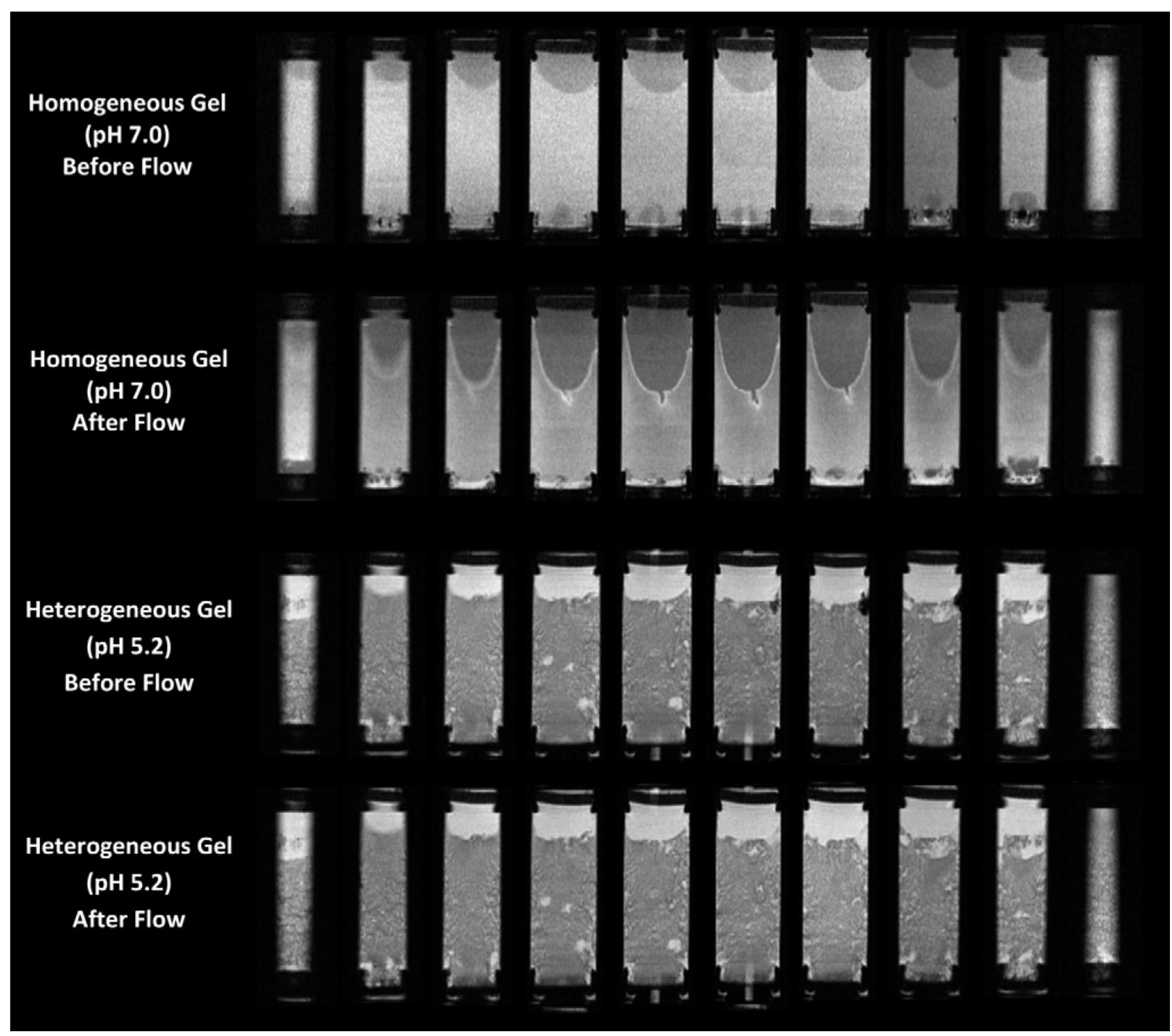

Fig. 2. NMR images show a series of eight $1 \mathrm{~mm}$ slices through the LC column showing a homogeneous $p H=7.0$ (top) and heterogeneous $p \mathrm{H}=5.2$ (bottom) gel before and after a full experimental run. For the $p \mathrm{H}=7.0$ gel the lighter colored regions indicate the location of the gel, while for the $p \mathrm{H}=5.2$ gel this is the water region and the gel regions are darker. This difference is due to different repetition times for the experiments $(1 \mathrm{~s} v s .5 \mathrm{~s})$ which result in different relaxation time weighting for each gel yet providing optimal contrast to observe differences in homogeneity and gel compression after flow. NMR experimental parameters: $256 \times 256$ pixels, 1 Average, SW: $50 \mathrm{kHz}, T_{R}: 1 \mathrm{~s}$ or $5 \mathrm{~s}, T_{E}: 40 \mathrm{~ms}, 1 \mathrm{~mm}$ slice, FOV: $30 \mathrm{~mm} \times 12 \mathrm{~mm}$, Voxel Resolution: $117 \times 47 \times 1000 \mu \mathrm{m}^{3}$.

where $E=\frac{S(g)}{S(0)}$ normalizes for influences from NMR relaxation effects, $\boldsymbol{q}=\frac{\gamma \delta \boldsymbol{g}}{2 \pi}$ is the reciprocal wavelength vector and $\boldsymbol{R}=\boldsymbol{r}^{\prime}-\boldsymbol{r}$ is the dynamic displacement. The propagator $P\left(\boldsymbol{r} \mid \boldsymbol{r}^{\prime}, \Delta\right)$, or Van Hove self-correlation function, is the conditional probability that a molecule starting at position $\boldsymbol{r}$ will move to the position $\boldsymbol{r}^{\prime}=\boldsymbol{r}+\boldsymbol{R}$ over a time period $\Delta$, whilst the average propagator $\bar{P}(\boldsymbol{R}, \Delta)$ is defined as the average over the initial molecular distribution $\rho(\boldsymbol{r})$ and this averaged propagator of molecular displacement $\boldsymbol{R}$ over observation time $\Delta$ is obtained directly from a Fourier transform of $E(\boldsymbol{q}, \Delta)$ with respect to $\boldsymbol{q}$.
In the case of diffusion $D$ (incoherent motion) superimposed on convective velocity $v$ (coherent motion) the result is,

$$
\bar{P}(\boldsymbol{R}, \Delta)=\frac{1}{2 \sqrt{\pi D \Delta}} \exp \left[-\frac{(\boldsymbol{R}-\boldsymbol{v} \Delta)^{2}}{4 D \Delta}\right] .
$$

The normalised echo signal decays exponentially, characterized by a purely diffusive coefficient $D$

$$
E(\boldsymbol{q})=\exp \left(-D(2 \pi \boldsymbol{q})^{2} \Delta\right) \cdot \exp (i 2 \pi \boldsymbol{q} \cdot\langle v\rangle \Delta) .
$$

This well-known Stejskal-Tanner equation [56] results in a measure of the effective diffusion coefficient of the sample. 


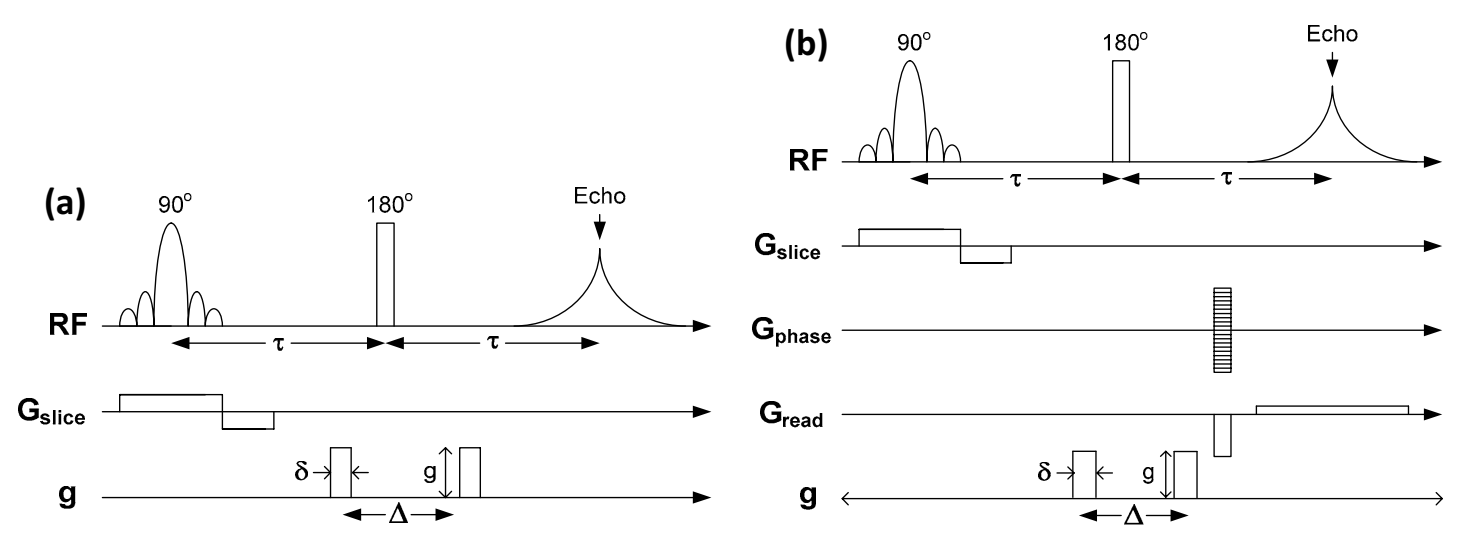

Fig. 3. (a) Slice selective pulsed gradient spin echo (PGSE) sequence to measure spin displacement. $\tau$ is the echo time, $\Delta$ is the measurement time, $\delta$ and $\boldsymbol{g}$ are the duration and magnitude of the pulsed field gradients. (b) Slice selective velocity map pulse sequence.

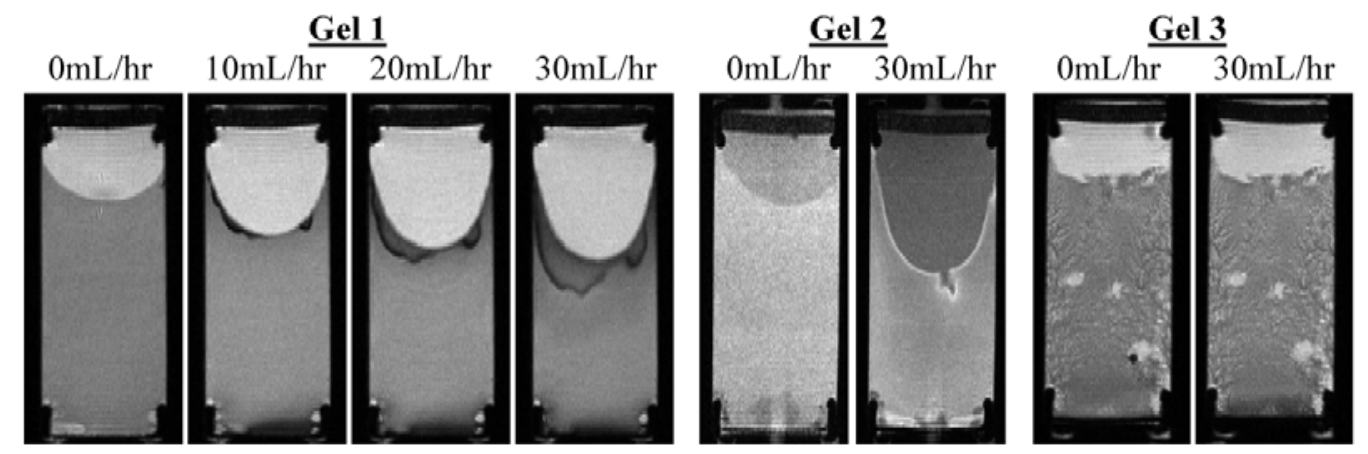

Fig. 4. NMR images of three gels after flow ceases for the indicated volumetric flow rates. Gel 1 : homogeneous $p H=7.0$ gel after the flow of salt solution $(0.3 \mathrm{M} \mathrm{NaCl})$. Gel 2: homogeneous $p \mathrm{H}=7.0$ gel after the flow of DI water. Gel 3: heterogeneous $p \mathrm{H}=5.2$ gel after the flow of DI water. NMR experimental parameters: $256 \times 256$ pixels, 1 Average, $\mathrm{SW}: 50 \mathrm{kHz}, T_{R}: 1 \mathrm{~s}$ or $5 \mathrm{~s}$, $T_{E}: 40 \mathrm{~ms}, 1 \mathrm{~mm}$ slice, FOV: $30 \mathrm{~mm} \times 12 \mathrm{~mm}$, Voxel Resolution: $117 \times 47 \times 1000 \mu \mathrm{m}^{3}$. By measuring the lowest point of the gel the following strains are measured for each flow rate of the homogeneous gels with salt: $\Delta L / L=0.00,0.21,0.27$ and 0.31 and without salt: $\Delta L / L=0.00$ and 0.31 . No strain is observed for the heterogeneous gel.

The $\boldsymbol{q}$-space pulse sequences presented above to measure spin displacement can be combined with NMR imaging $(\boldsymbol{k}$ space) methods to obtain an image where each pixel has $\boldsymbol{q}$-space information, see fig. $3 \mathrm{~b}$, allowing for the imaging of coherent motion from the phase shift of the signal known as a velocity map $[33,57]$.

\section{Results and discussion}

\subsection{Imaging structure and velocity}

Several samples were made with a $p \mathrm{H}=5.2$ to produce a heterogeneous gel and several samples were prepared at a $p \mathrm{H}=7.0$ to produce a homogeneous gel. Figure 1 shows NMR images of two representative gel samples used in this study before and after flow experiments as well as velocity maps during flow. For comparison, stereo microscope and compound microscope images of the same gels sliced in the middle are also shown, the gels have been stained with a crystal violet dye. It is clear that the $p \mathrm{H}=7.0$ gel is homogeneous on the macroscopic scale $(\geq 100 \mu \mathrm{m})$ while the $p \mathrm{H}=5.2$ gel is heterogeneous on the macroscopic scale. These differences are due to the microscopic structure of the proteins as they denature and aggregate during the preparation of the gels.

On the $(\mu \mathrm{m})$ scale both gels have some heterogeneity, with the homogeneous gel having much smaller gaps between aggregates (fig. 1e). The velocity maps are particularly illuminating, they are averaged over a $1 \mathrm{~mm}$ slice with an in-plane resolution of $47 \times 47 \mu \mathrm{m}^{2}$ and show clear signs of flow heterogeneity within the $\beta$-lactoglobulin gel formed at $p \mathrm{H}=5.2$, while only minimal flow heterogeneity exists within the $\beta$-lactoglobulin gel formed at $p \mathrm{H}=7.0$. It is evident, fig. 1 top row, that the heterogeneous gel has many openings in the gel structure on the order of 100 $1000 \mu \mathrm{m}$. This heterogeneity is expected to allow significant backbone flow, similar to percolation structures [58], across the gel during flow experiments. This explains the ability of the heterogeneous gel to resist structural deformation, even at high flow rates $(100 \mathrm{~mL} / \mathrm{h})$, due to the lower pressure gradient required to push the solvent 

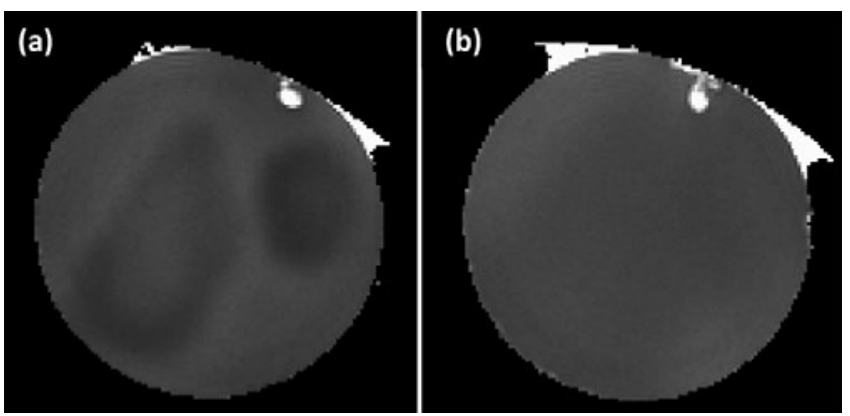

Fig. 5. $T_{2}$ relaxation map for the homogeneous gel after flow experiments, to investigate the cause of the contrast in the NMR images (fig. 4) when $0.3 \mathrm{M} \mathrm{NaCl}$ is flowed through the gel. $1 \mathrm{~mm}$ slices of the excitation region (a) and $2 \mathrm{~mm}$ down steam (b) from this region. $T_{2}$,long gel $\sim 0.2 T_{2}$, water,$T_{2}$,short gel $\sim$ $0.81 T_{2}$, long gel. NMR experiment parameters: $256 \times 256$ pixels, 16 Average, SW: $50 \mathrm{kHz}, T_{R}: 5 \mathrm{~s}, T_{E}: 12 \mathrm{~ms}, 16$ echos, $10 \times$ $1 \mathrm{~mm}$ slices, FOV: $12 \mathrm{~mm} \times 12 \mathrm{~mm}$, Voxel Resolution: $47 \times 47 \times$ $1000 \mu \mathrm{m}^{3}$.

molecules through the gel at fixed volumetric flow rate. This is contrasted by the homogeneous gel which on the scales $>O(50 \mu \mathrm{m})$ is homogeneous, fig. 1. This is important because at the shortest experimental time of this study $(\Delta=25 \mathrm{~ms})$ the standard deviation $(\sigma)$ for the displacement of water molecules due to free diffusion, i.e. the diffusive length $l_{D} \sim \sqrt{2 D_{0} \Delta}$, is $10 \mu \mathrm{m}$, indicating that on this displacement scale there should be homogeneity in flow dynamics of solvent molecules across the gel, characterized by a Gaussian distribution of displacement during flow experiments.

Since there are no macro-scale openings for backbone flow development in the homogeneous gel, pressure gradients across this gel are much higher than those for the heterogeneous gel. This explains why this gel is compressed by flow, see figs. 2 and 4, and finally fractures for the flow of DI water once the gel structure has compressed/deformed sufficiently. To determine the impact of the presence of $\mathrm{NaCl}$ in the flowing solvent on the homogeneous gel elastic response and fracture $0.3 \mathrm{M} \mathrm{NaCl}$ is also used. As can be seen in fig. 4, a contrasting signal region forms in the image when a salt solution $(0.3 \mathrm{M} \mathrm{NaCl})$ is pumped through the homogeneous gel, which is not present when DI water without salt is the flowing solvent. This region propagates further into the gel with increasing flow rate. The elastic strain $\Delta L / L$ for the DI water and $0.3 \mathrm{M} \mathrm{NaCl}$ solution are similar but the $\mathrm{NaCl}$ flow does not fracture the gel.

\subsection{Changes in $\mathrm{NMR}$ relaxation due to $\mathrm{NaCl}$ flow}

To investigate the origins of these phenomena, a $T_{2}$ relaxation experiment (fig. 5) was performed on the gel after flow experiments had been conducted. Spin-spin or $T_{2}$ magnetic relaxation provides information on the rotational mobility of the water molecules. In regions of greater gel polymer density the restricted slower rotational molecular motion decreases $T_{2}$ relative to more mobile water molecules in lower-polymer-density regions and results in relaxation-weighted contrast in the NMR image. The gel was removed from the LC column and placed inside a $20 \mathrm{~mm}$ O.D. glass vial. $T_{2}$ relaxation images are presented which show $1 \mathrm{~mm}$ slices of a region corresponding to the region that shows some increased relaxation weighted contrast (fig. 5a) and a region two millimeters downstream (fig. 5b), where no additional relaxation weighted contrast of the gel has occurred. A bright region of free water trapped by capillary force between the inner wall of the glass vial and the gel was used to obtain the $T_{2}$ relaxation time of free water $\left(T_{2}\right.$,water $)$. The longer $T_{2}$ regions of the gel have $T_{2}$, long gel $\sim 0.2 T_{2}$,water, while the shorter $T_{2}$ regions of the gel have $T_{2 \text {,short gel } \sim 0.81 T_{2} \text {, long gel }}$ $\left(\sim 0.16 T_{2, \text { water }}\right)$. This $20 \%$ lower $T_{2}$ indicates greater restriction of rotational mobility of the water molecules in the darker regions of the images. A potential NMR artifact related to the addition of salts, is the requirement of more rf power to cause the same angular spin magnetization flip. This rf pulse intensity variation would alter the relative image intensity of regions if salt were concentrating in the low-signal-intensity region. NMR imaging experiments were conducted to determine if this signal intensity variation was due to salt concentration. The $180^{\circ} \mathrm{rf}$ pulse strength was varied by $1 \mathrm{db}$ over a range of $10 \mathrm{db}$ above and below the correct $180^{\circ} \mathrm{rf}$ pulse. These experiments showed negligible signal intensity impact within experimental error, indicating that this lowering of pixel intensity in the NMR images is not due to higher local salt concentrations.

A potential explanation of the cause of the shortening of the $T_{2}$ in the gel may be found in the gel literature, where it has been shown that the effect of high salt concentrations on $\beta$-lactoglobulin during the aggregation process is to increase aggregate branching and cause an increase in local protein density. At salt concentrations higher than $0.1 \mathrm{M}$ the critical association concentration (CAC) drops to less than or equal to $0.1 \mathrm{~g} / \mathrm{L}$, while the gelation concentration $\left(C_{g}\right)$ equals $1 \mathrm{~g} / \mathrm{L}$ at $0.4 \mathrm{M} \mathrm{NaCl}$ [25]. For the current experiments $C=90 \mathrm{~g} / \mathrm{L}$ and therefore $C \gg \mathrm{CAC}$ and $C_{g}$ so the activation energy for aggregation is low, indicating that the pressure-driven flow introduces sufficient energy and contact between aggregates at high-pressure regions to cause local densification of gel aggregates in the presence of salt. These denser regions are distinguishable by NMR (fig. 4) as the flow rate and hence strain increases. These regions of local gel density increase correlate to the local stress and serve as indicators for submicron gel structural changes and as location predictors for macroscopic fracture.

\subsection{Flow dynamics by NMR}

NMR flow dynamics experiments were conducted for both the homogeneous and heterogeneous gels at flow rates of 0, 10, 20 and $30 \mathrm{~mL} / \mathrm{h}$. Before examining the data, it is important to clarify the relative scales of displacement due to advection and diffusion in the two gels. The flow rates 10, 20 and $30 \mathrm{~mL} / \mathrm{h}$ correspond to mean solvent velocities of $35.4 \mu \mathrm{m} / \mathrm{s}, 70.7 \mu \mathrm{m} / \mathrm{s}$ and $106.1 \mu \mathrm{m} / \mathrm{s}$ based on the volumetric flow divided by the cross-sectional area of the 

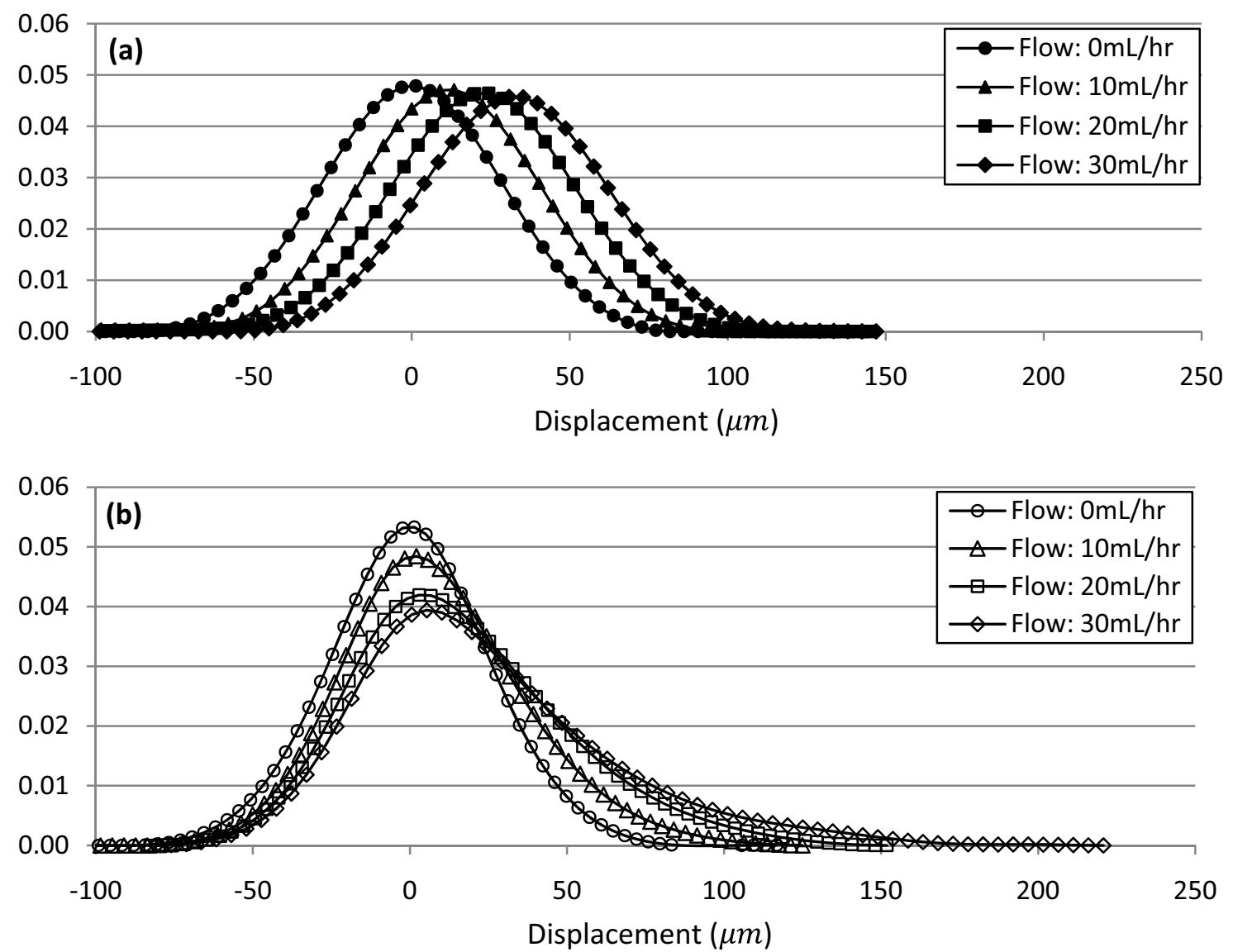

Fig. 6. Propagators for the flow of DI water through the homogeneous (a) and heterogeneous (b) gels in the parallel-to-flow direction at increasing flow rate for $\Delta=200 \mathrm{~ms}$.

LC column. For the range of experimental displacement observation times used in this study, $\Delta=0.025-0.600 \mathrm{~s}$, the advective mean displacements based on the superficial velocity range from $0.9-63.7 \mu \mathrm{m}$, scaling linearly with $\Delta$. The standard deviation $(\sigma)$ for the displacement due to the free diffusion of water at $20^{\circ} \mathrm{C}$ on the same time scale, $\Delta=0.025-0.600 \mathrm{~s}$, is $10-49 \mu \mathrm{m}$, where the displacement scales as $\sqrt{\Delta}$. Since the variance of displacement is affected by both these processes, it is expected that unless significant advective driven hydrodynamic dispersion occurs due to mechanical, Saffman and Taylor dispersion processes, the overwhelming contribution will be due to the diffusion of the solvent molecules within the gel structure.

Propagator experiments showing the probability of displacement for the homogeneous and heterogeneous gels at $\Delta=200 \mathrm{~ms}$, for the flow rates of $0,10,20$ and $30 \mathrm{~mL} / \mathrm{h}$ are shown in fig. 6. For the homogeneous gel (fig. 6a), the probability distribution is Gaussian and changes minimally with increasing flow rate, a simple broadening and a mean displacement shift is all that is observed. As will be seen below, the width of the Gaussian distribution changes with increasing flow rate due to the impact of advective motion. The Gaussian dynamics for the homogeneous gel is contrasted by the flow through the heterogeneous gel (fig. 6b) which shows a significant change in the probability distribution of displacement with increasing flow rate. The data show a transition towards a non-Gaussian probability distribution with a significant long displacement, backbone-flow type, contribution. A simple reduction of accessible pore space for flow would result in a Gaussian shape shifted to larger displacements, while a broad distribution of dynamics with a tail of higher probability of large displacements clearly indicates regions of small and large displacements in backbone or channeling flows.

To compare the propagator data the variance of the propagator for both gels and all flow rates and experimental times investigated are presented in fig. 7 . The standard statistical definitions of mean (1st raw moment) $\mu=\sum_{i} x_{i} P\left(x_{i}\right)$ and variance (2nd central moment) $\sigma^{2}=$ $\sum_{i}\left(x_{i}-\mu\right)^{2} P\left(x_{i}\right)$ of the normalized propagator are used.

\subsection{Homogeneous gel}

In the parallel-to-flow direction for the homogeneous gel, for the first three flow rates $(0 \mathrm{~mL} / \mathrm{h}, 10 \mathrm{~mL} / \mathrm{h}$ and $20 \mathrm{~mL} / \mathrm{h}$ ) the variance is linear with time. The dispersion at $10 \mathrm{~mL} / \mathrm{h}$ is systematically lower than the pure molecu- 

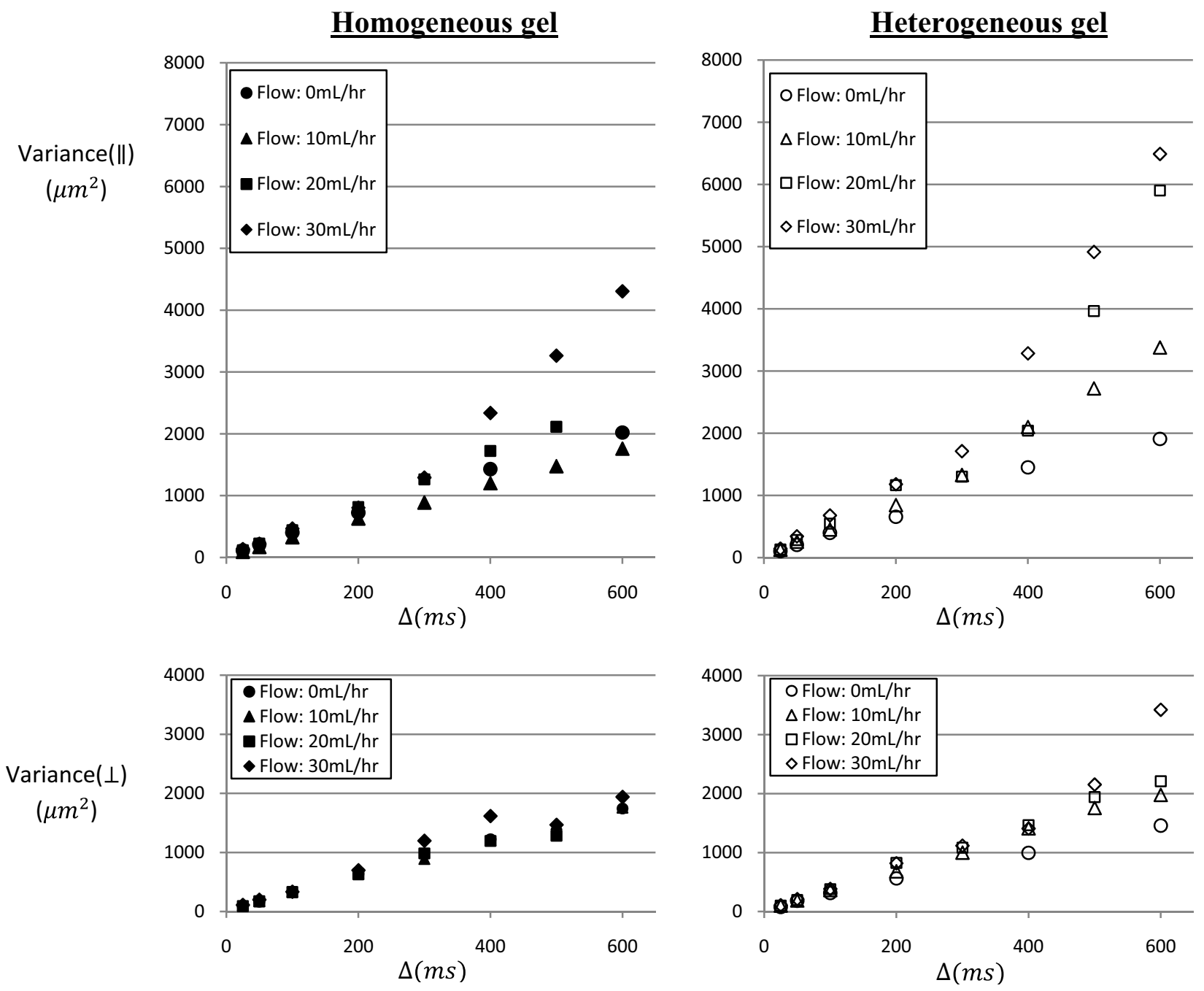

Fig. 7. Variance obtained from the probability distributions obtained using propagator experiments for the homogeneous (filled symbols) and heterogeneous (open symbols) gels at $0,10,20$ and $30 \mathrm{~mL} / \mathrm{h}$, in directions parallel $(\|)$ and perpendicular $(\perp)$ to the axial flow direction.

lar diffusion at $0 \mathrm{~mL} / \mathrm{h}$, while the $20 \mathrm{~mL} / \mathrm{h}$ flow is higher. At a flow rate of $30 \mathrm{~mL} / \mathrm{h}$, the variance follows the variance for $20 \mathrm{~mL} / \mathrm{h}$ up till $\Delta=300 \mathrm{~ms}$, and then deviates significantly with much higher dispersion at $\Delta \geq 400 \mathrm{~ms}$. This can be clearly seen by comparison of the propagators for each flow rate at fixed displacement observation times shown in fig. 8. In the perpendicular-to-flow direction for the homogeneous gel, the variance for the first three flow rates $(0 \mathrm{~mL} / \mathrm{h}, 10 \mathrm{~mL} / \mathrm{h}$ and $20 \mathrm{~mL} / \mathrm{h})$ is approximately the same over the whole range of $\Delta$, while for $30 \mathrm{~mL} / \mathrm{h}$ it varies but trends with the lower flow rate data. An important aspect of the transport in the homogeneous gel is that the gel is compressing as the flow rate increases (fig. 4) and at the $30 \mathrm{~mL} / \mathrm{h}$ flow rate, the shorter $T_{2}$ gel region (discussed above) has reached the slice being excited for the propagator experiments. Therefore the highest-flow-rate data $(30 \mathrm{~mL} / \mathrm{h})$ deviation from the lower-flow-rate data trends is due to the gel structural changes directly affecting the measured dynamics. This compression also explains the hydrodynamic dispersion at $10 \mathrm{~mL} / \mathrm{h}$ being lower than the dispersion at $0 \mathrm{~mL} / \mathrm{h}$, since the diffusion contribution to the dispersion has decreased due to increased restriction, which is not offset by the hydrodynamic dispersion due to the advective dynamics since at this flow rate the diffusive length scale is larger than the advective length scale. Since there is minimal contribution due to advection-induced hydrodynamic dispersion in the perpendicular-to-flow direction the only data which deviates significantly is for $30 \mathrm{~mL} / \mathrm{h}$, while the $10 \mathrm{~mL} / \mathrm{h}$ data is only slightly lower on average than the $0 \mathrm{~mL} / \mathrm{h}$ data.

\subsection{Heterogeneous gel}

In the parallel-to-flow and perpendicular-to-flow directions for the heterogeneous gel, the variance follows the general trend of increasing variance with increasing flow rate at the same experimental time $(\Delta)$. An important result is the large change in the slope of the variance as 


\section{$\underline{\text { Homogeneous gel, parallel-to-flow direction }}$}
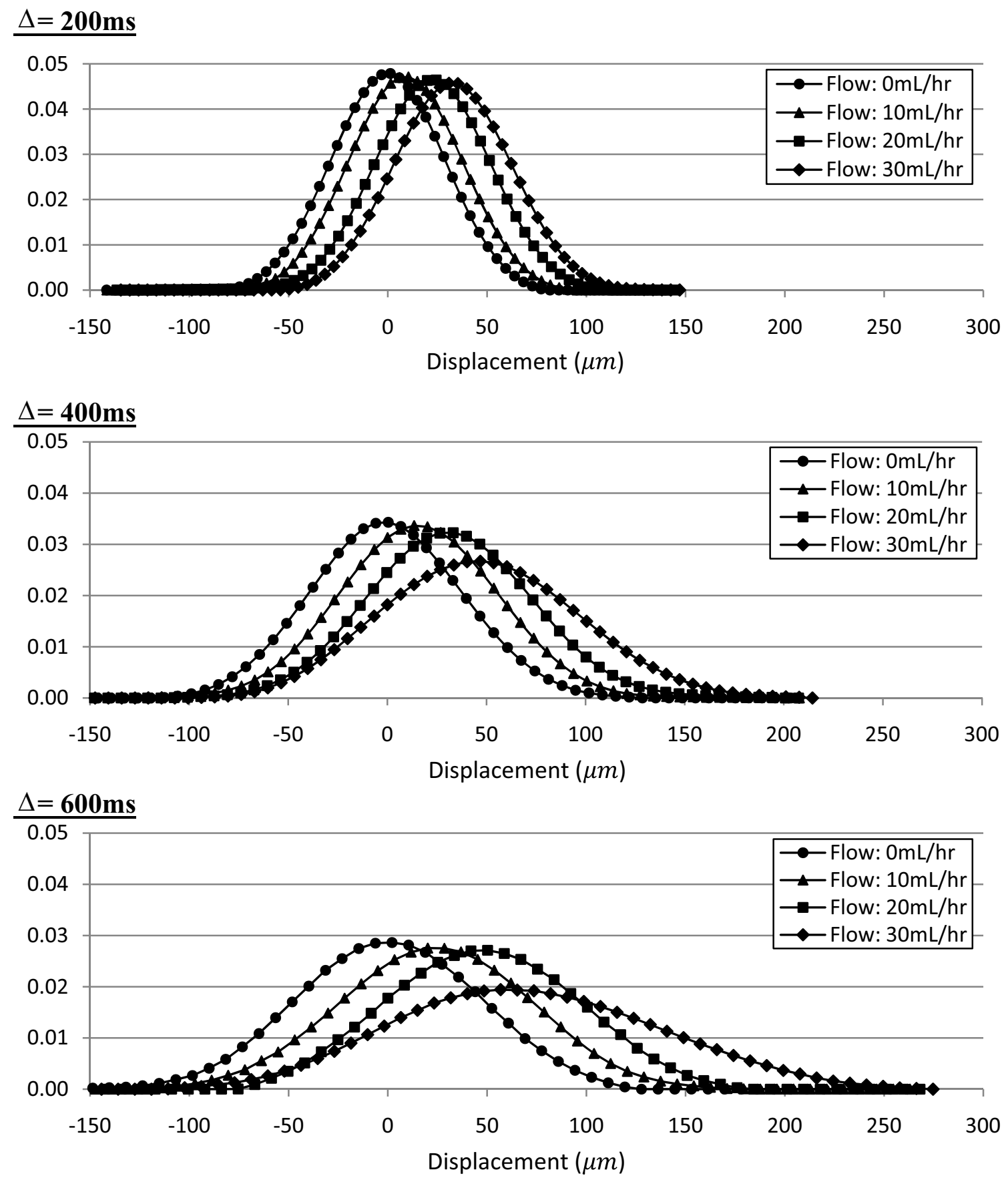

Fig. 8. Shows the evolution of the propagators with flow rate at different experimental times $(\Delta=200,400$ and $600 \mathrm{~ms})$ for the homogeneous gel.

a function of $\Delta$ seen at $\Delta>300 \mathrm{~ms}$ for both the flows at $20 \mathrm{~mL} / \mathrm{h}$ and $30 \mathrm{~mL} / \mathrm{h}$. This is associated with a significant growth in a long displacement (backbone flow) tail in the probability distribution of displacement, evident in fig. 9 .

Figure 9 shows another interesting trend in the heterogeneous gel propagators, which is the initial shift in the peak of the probability distribution towards higher mean displacements as the flow increases, which then ceases at long experimental times $(\Delta=400$ and $600 \mathrm{~ms})$ and high flow rates $(20 \mathrm{~mL} / \mathrm{h}$ and $30 \mathrm{~mL} / \mathrm{h})$. This behavior is indicative of a hold-up-like effect whereby solvent molecules are trapped in non-advective regions [59].

This behavior can be explained by consideration of a two-population model. One population of solvent 


\section{Heterogeneous gel, parallel-to-flow direction}
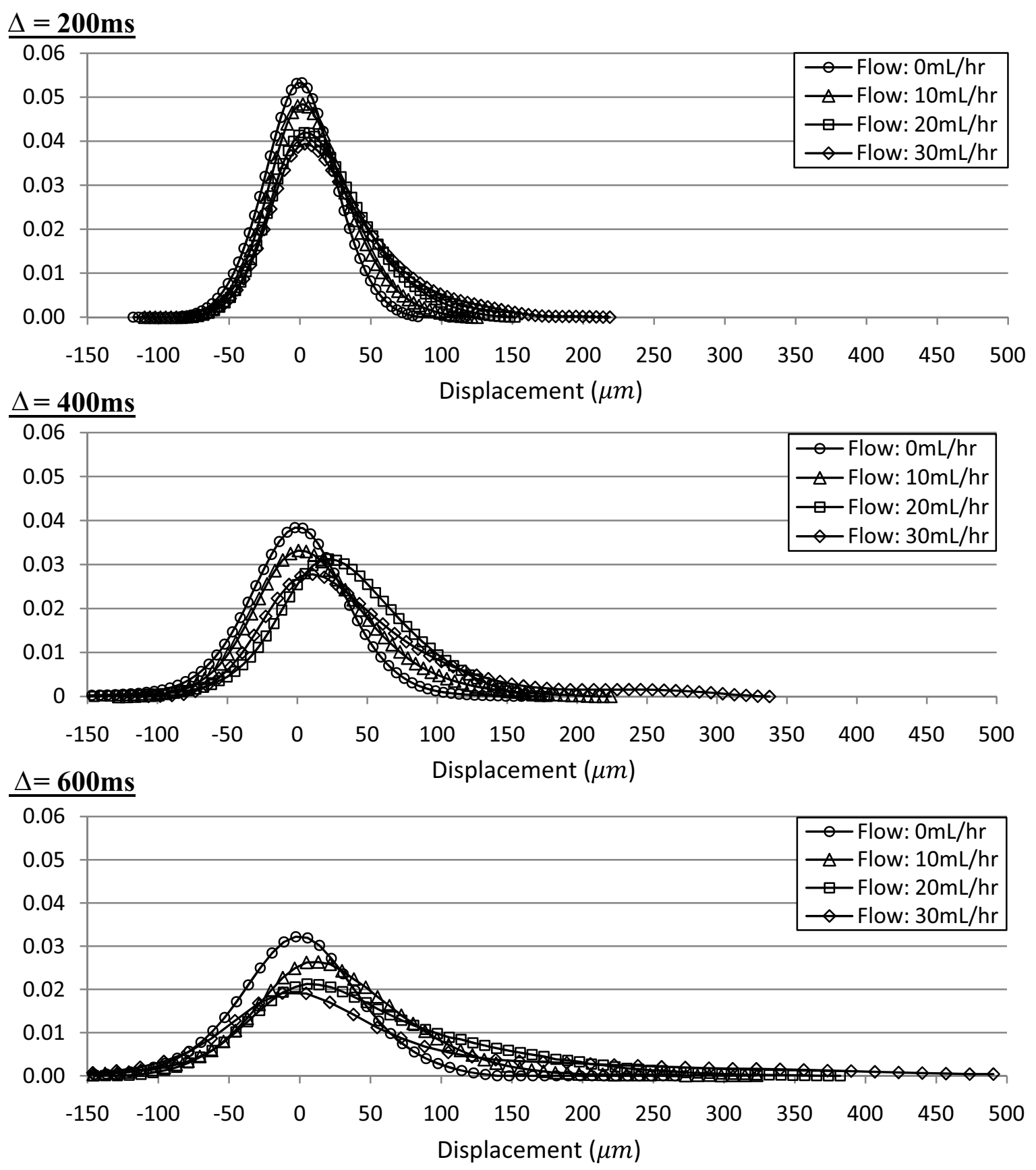

Fig. 9. Shows the evolution of the propagators with flow rate at different experimental times $(\Delta=200,400$ and $600 \mathrm{~ms})$ for the heterogeneous gel.

molecules behaves as in the homogeneous gel, i.e. has a Gaussian-like distribution of displacement shifting to higher mean displacements with increasing flow rates. The solvent molecules of the second population occupy the fast flow regions, seen clearly in the velocity map shown in fig. 1. The solvent molecules can move between these transport regions by molecular diffusion, with an increased probability of having occupied both regions as $\Delta$ increases.
However as the mean displacement of the molecules in the slow transport region increases, more and more molecules will also experience the fast flow region, with a displacement of $\sim 25 \mu \mathrm{m}$ indicating an approximate limit as to how far the molecules can displace without experiencing the fast flow regime. The $\sim 25 \mu \mathrm{m}$ length is obtained from the cross-over point of the probability distribution functions at $\Delta=200 \mathrm{~ms}$. 


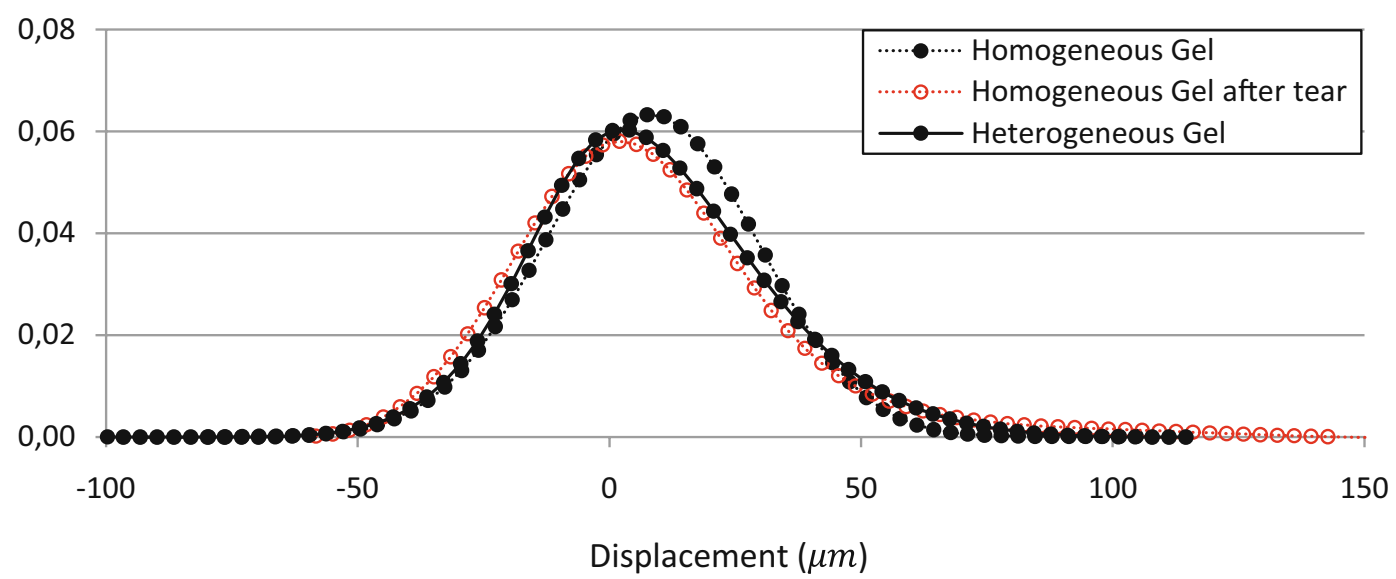

Fig. 10. Propagators for the homogeneous gel before and after tearing and the heterogeneous gel at a flow rate of $20 \mathrm{~mL} / \mathrm{h}$ for $\Delta=100 \mathrm{~ms}$, parallel-to-flow direction.

Table 1. Mean velocities in the parallel-to-flow $\left\langle v_{\|}\right\rangle$and perpendicular-to-flow $\left\langle v_{\perp}\right\rangle$ directions at flow rates of $0,10,20$ and $30 \mathrm{~mL} / \mathrm{h}$ obtained from propagator experiments by using $\left\langle v_{\| \text {or } \perp}\right\rangle=\langle\mu\rangle / \Delta$.

\begin{tabular}{|c|c|c|c|c|}
\hline & \multicolumn{2}{|c|}{$\left\langle v_{\|}\right\rangle(\mu \mathrm{m} / \mathrm{s})$} & \multicolumn{2}{c|}{$\left\langle v_{\perp}\right\rangle(\mu \mathrm{m} / \mathrm{s})$} \\
\hline $\begin{array}{c}\text { Flow rate } \\
(\mathrm{mL} / \mathrm{h})\end{array}$ & Hom. gel & Het. gel & Hom. gel & Het. gel \\
\hline 0 & 0 & 0 & -1 & 2 \\
\hline 10 & 44 & 43 & 5 & 4 \\
20 & 89 & 88 & 5 & 6 \\
30 & 130 & 129 & 22 & 10 \\
\hline
\end{tabular}

\subsection{Gel tear impact on transport}

Figure 10 shows the propagators in the parallel-to-flow direction at a flow rate of $20 \mathrm{~mL} / \mathrm{h}$ at $\Delta=100 \mathrm{~ms}$ for the heterogeneous gel, the homogeneous gel and the homogeneous gel after a fracture has formed (see fig. 1 for NMR images of the fracture). The similarities in the propagators for the heterogeneous gel and the homogeneous gel after tearing are striking considering how dissimilar in structure and dynamics the two gels are. After fracture a preferential flow path has been created where the two-population model is clearly applicable, even though the gel is still homogeneous and the fracture does not permeate the entire gel. The backbone flow is likely to be a result of a non-uniform distribution of initial velocities set-up by the deformed and torn front.

\subsection{Transport analysis}

The mean displacement $(\langle\boldsymbol{R}\rangle)$ measured in the propagator experiments can be used to quantify the mean solvent velocity measured in the parallel-to-flow $\left\langle v_{\|}\right\rangle$and perpendicular-to-flow $\left\langle v_{\perp}\right\rangle$ directions by using $\left\langle v_{\| \text {or } \perp}\right\rangle=$ $\frac{\langle\boldsymbol{R}\rangle_{\| \text {or } \perp}}{\Delta}$, see table 1 .
Table 2. Excluded-volume fraction $\left(\phi_{E}\right)$ obtained from $\phi_{E}=$ $1-\left\langle v_{z}\right\rangle /\left\langle v_{\|}\right\rangle$, where $\left\langle v_{z}\right\rangle$ is the superficial velocities for flow rates 10,20 and $30 \mathrm{~mL} / \mathrm{h}$, respectively $\left\langle v_{z}\right\rangle=35.4,70.7$ and $106.1 \mu \mathrm{m} / \mathrm{s}$.

\begin{tabular}{|c|c|c|}
\hline & \multicolumn{2}{|c|}{ Excluded-volume fraction $\left(\phi_{E}\right)$} \\
\hline $\begin{array}{c}\text { Flow rate } \\
(\mathrm{mL} / \mathrm{h})\end{array}$ & Hom. gel & Het. gel \\
\hline 10 & 0.195 & 0.177 \\
20 & 0.204 & 0.195 \\
30 & 0.183 & 0.177 \\
\hline Avg $\pm 3 \mathrm{SD}$ & $0.194 \pm 0.033$ & $0.183 \pm 0.030$ \\
\hline
\end{tabular}

It is important to note the mass conservation does not restrict the perpendicular (radial) velocity component to be zero if the excited volume (slice) of fluid moves through a radially asymmetric flow field. For the gels with multiple radially asymmetric channels many reasonable geometries exist in which a non-zero local radial velocity emerges.

Since the perpendicular velocity component grows with increasing flow rate (i.e. gel compression) it indicates that the compression of the gel increases the asymmetry in the radial component of the flow field. Good evidence for compression asymmetry comes from the shorter $T_{2}$ region in fig. $5 \mathrm{a}$, which is not symmetric in the radial direction. The results in table 1 indicate that the compression of the gel is causing the growth of flow field asymmetry or heterogeneity.

The measured velocity can also be used to determine the excluded-volume fraction by using $\phi_{E}=1-\frac{\left\langle v_{z}\right\rangle}{\left\langle v_{\|}\right\rangle}$, where $\left\langle v_{z}\right\rangle$ is the velocity through the column with no gel which is, respectively, 35.4, 70.7 and $106.1 \mu \mathrm{m} / \mathrm{s}$ for flow rates 10,20 and $30 \mathrm{~mL} / \mathrm{h}$, see table 2 . These calculations indicate $\phi_{E}=0.194 \pm 0.033$ and $0.183 \pm 0.030$ for the homogeneous and heterogeneous gels, respectively. The $\beta$-lactoglobulin weight fraction added to form the gel was 0.09 . 
Homogeneous gel
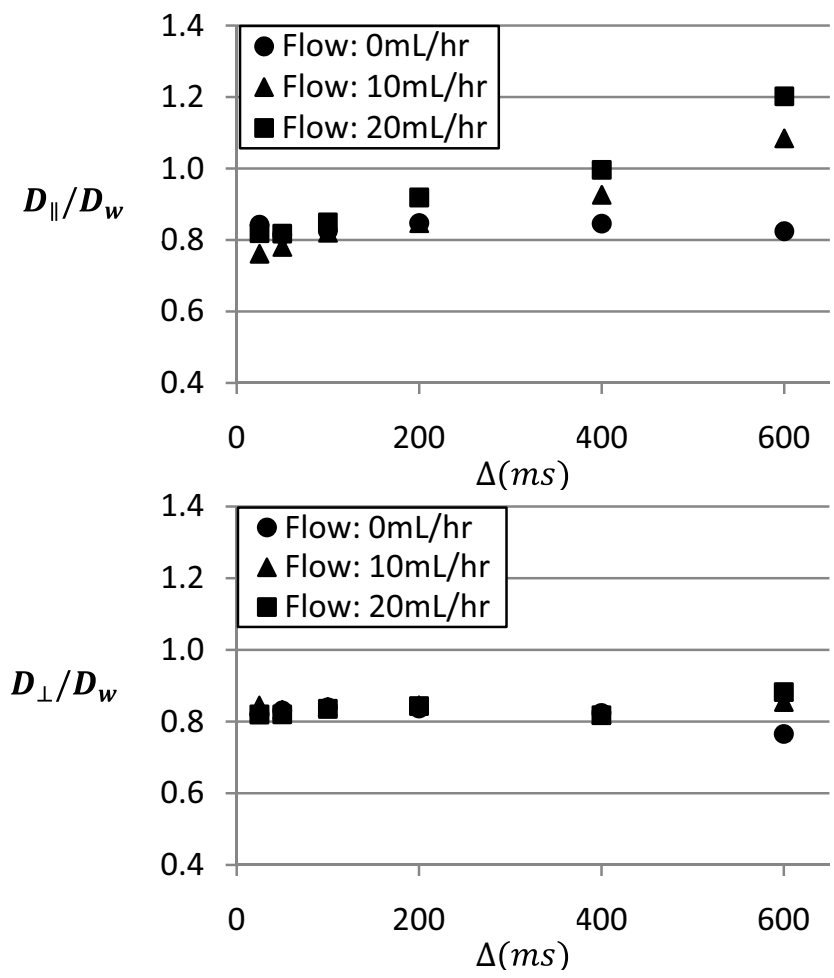

Heterogeneous gel
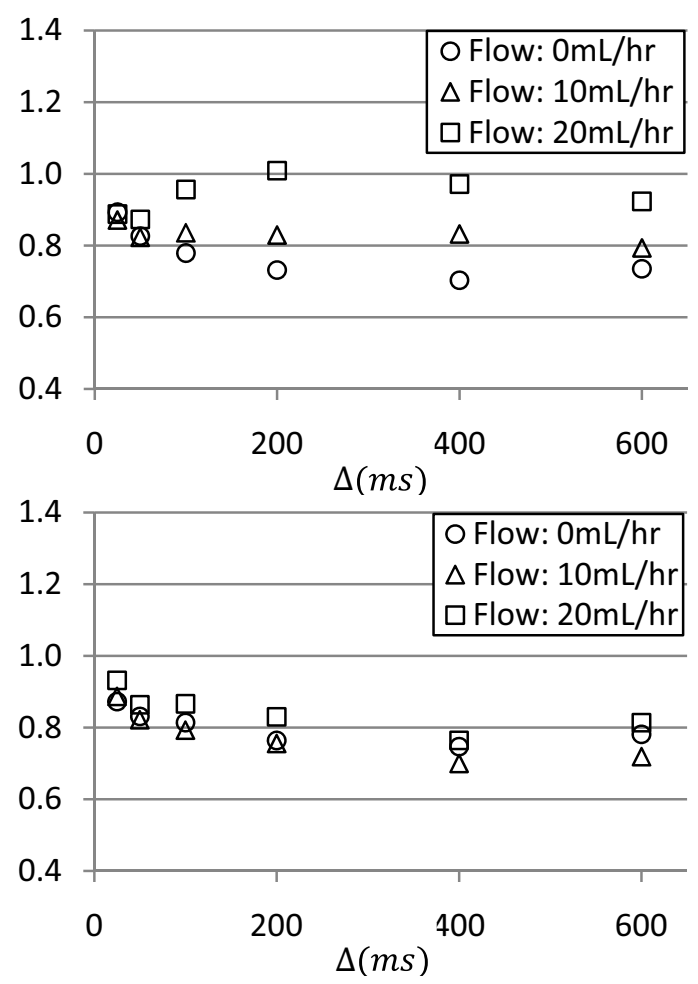

Fig. 11. Shows the normalized effective diffusion coefficients as a function of time $(\Delta)$ for the homogeneous (closed symbols) and heterogenous gels (open symbols) at different flow rates $(0 \mathrm{~mL} / \mathrm{h}, 10 \mathrm{~mL} / \mathrm{h}$ and $20 \mathrm{~mL} / \mathrm{h}$ ). NMR experimental parameters: Acquisition size: $256,32 \boldsymbol{q}$-points, $\boldsymbol{g}_{\max }: 0.344-0.129 \mathrm{~T} / \mathrm{m}$ for $\Delta=25-600 \mathrm{~ms}, \delta=4 \mathrm{~ms}, \mathrm{SW}: 5 \mathrm{kHz}, N_{\mathrm{avg}}: 8, \Delta Z: 1 \mathrm{~mm}$.

Table 3. Stretched exponential fits $(\beta)$ to the low- $\boldsymbol{q}$ data for flow through the homogeneous and heterogeneous gels at $\Delta=$ 25, 100 and $400 \mathrm{~ms}$, for flow rates $0,10,20$ and $30 \mathrm{~mL} / \mathrm{h}$, in the parallel-to-flow direction $(Z)$. Note $(* * *)$ refers to lack of sufficient low- $\boldsymbol{q}$ points for an accurate fit.

\begin{tabular}{|c|c|c|c|c|}
\hline & & & Hom. gel & Het. gel \\
\hline$\Delta(\mathrm{ms})$ & $\begin{array}{c}\text { Flow rate } \\
(\mathrm{mL} / \mathrm{h})\end{array}$ & Dir. & $\beta$ & $\beta$ \\
\hline 25 & 0 & $Z$ & 1.85 & 1.67 \\
100 & 0 & $Z$ & 1.80 & 1.67 \\
400 & 0 & $Z$ & 1.83 & 1.69 \\
\hline 25 & 10 & $Z$ & 1.89 & 1.82 \\
100 & 10 & $Z$ & 1.80 & 1.69 \\
400 & 10 & $Z$ & 1.63 & 1.72 \\
\hline 25 & 20 & $Z$ & 1.82 & 1.75 \\
100 & 20 & $Z$ & 1.80 & 1.49 \\
400 & 20 & $Z$ & 1.65 & 1.41 \\
\hline 25 & 30 & $Z$ & 1.86 & $* * *$ \\
\cline { 4 - 5 } 100 & 30 & $Z$ & 1.87 & $* * *$ \\
\cline { 3 - 5 } 400 & 30 & $Z$ & 1.89 & $* * *$ \\
\hline
\end{tabular}

A useful method to further analyze the propagator data is to look at the stretched exponential wavelength scaling: $E(\boldsymbol{q}, \Delta) \sim \exp \left(-c \boldsymbol{q}^{\beta}\right)$ which corresponds to a space fractional ADE. The results of fitting the low- $\boldsymbol{q}$ space data for flow rates $0-30 \mathrm{~mL} / \mathrm{h}$ in the parallel-to-flow direction is shown in table 3 . These indicate that there is some restriction to the free diffusion of the homogeneous and heterogeneous gels $(\beta<2)$ and that the heterogeneous gel has a more exponential probability distribution than the homogeneous gel as water flows through the systems.

To further examine the transport behavior inside the different gels, the dispersion coefficient $\left(D^{*}\right)$ was measured using low- $\boldsymbol{q}$ experiments and Stejskal-Tanner plots [33]. Experiments were performed while varying the measurement time $(\Delta)$ and the flow rate both in the parallel-toflow and perpendicular-to-flow directions, see fig. 11. The dispersion coefficients in the parallel- and perpendicularto-flow direction were normalized using the free diffusion of water at $20^{\circ} \mathrm{C}, D_{w}=2 \times 10^{-9} \mathrm{~m}^{2} / \mathrm{s}$, to allow for a clear presentation of the dispersion relative to free diffusion and an easier comparison with the variance data. When comparing the normalized dispersion coefficient and the variance data it is important to emphasize that the former is obtained from the low- $\boldsymbol{q}$ data, $E(\boldsymbol{q}, \Delta)=1.0-0.7$, which corresponds to large displacements, i.e. signal effects due 


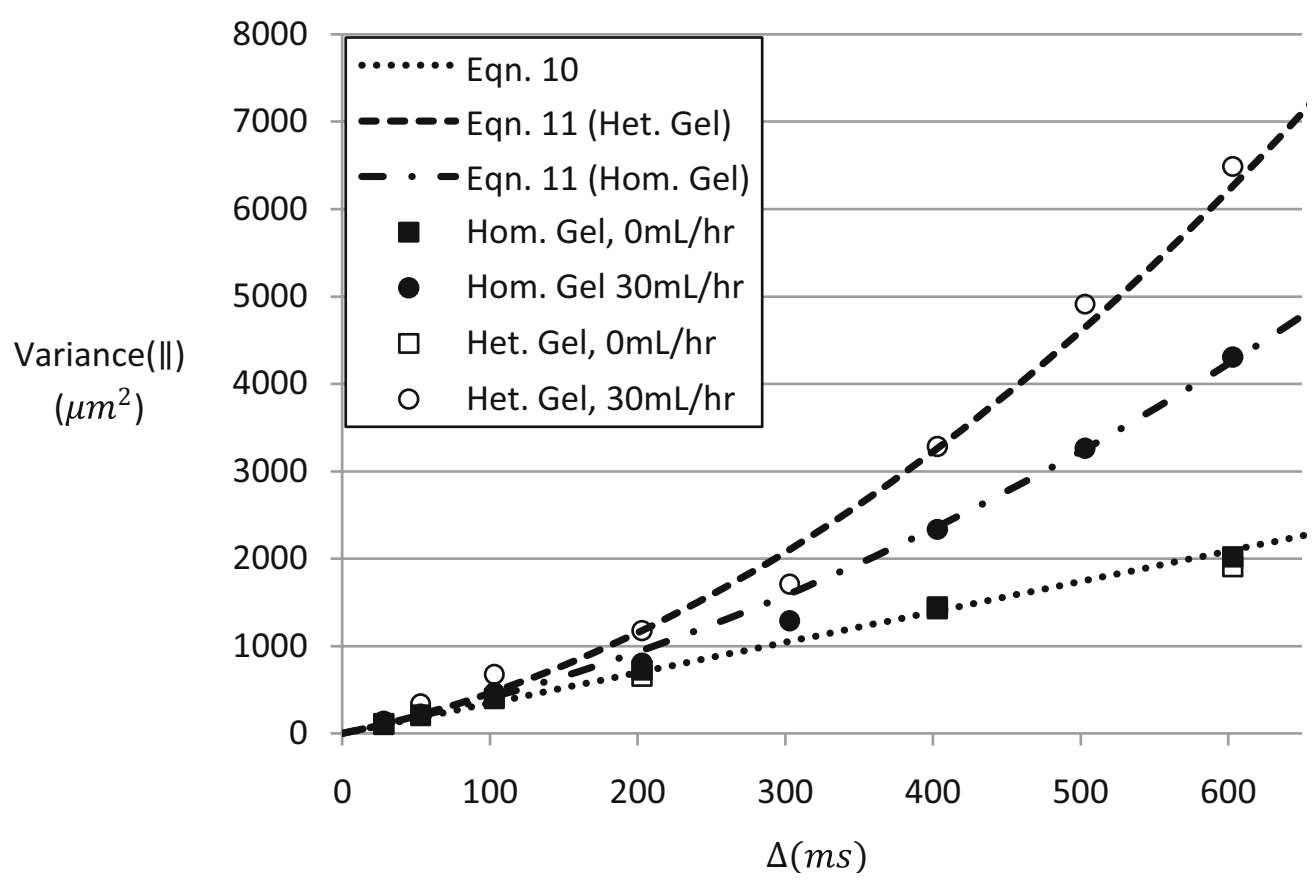

Fig. 12. Equations (10) and (11) fit to the variance obtained from propagator experiments for parallel-to-flow direction at $0 \mathrm{~mL} / \mathrm{h}$ and $30 \mathrm{~mL} / \mathrm{h}$ for the homogeneous and heterogeneous gels. The power for $\Delta^{\alpha}$ in eq. (11) is $\alpha=2$ for the heterogeneous gel and $\alpha=1.86$ for the homogeneous gel, while $K_{\alpha}=\left\langle U^{2}\right\rangle$.

to structures of lengths $O(50 \mu \mathrm{m})$ and larger affect the experiment, while the propagator is acquired from the entire $\boldsymbol{q}$-space data and therefore measures all displacements.

A simple restricted dynamics relationship is assumed, where the solvent molecules have all experienced a similar motional restriction due to the gel phase length scale $a$ such that $\Delta \gg \tau$, where $\tau \sim \frac{a^{2}}{D_{0}}$ is the correlation time of the solvent structure interaction. The variance

$$
\left\langle\boldsymbol{x}^{2}(\Delta)\right\rangle=2 D_{R} \Delta,
$$

where $D_{w}>D_{R}$ and $D_{R}$ is the restricted solvent molecule diffusion coefficient. It can be seen that this relationship models the correct linear relationship of variance with time for the no flow data for the homogeneous gel, where $D_{\|} / D_{w}$ and $D_{\perp} / D_{w}$ are approximately constant while the variance (fig. 7) has a linear dependence on $\Delta$. This relationship also agrees with the perpendicularto-flow direction data for the homogeneous gel. For the heterogeneous gel, the data is affected by the restriction length scale, since a larger diffusive length scale is present $\left(\tau \sim \frac{a^{2} \text { het }}{D_{0}} \gg \frac{a^{2} \text { hom }}{D_{0}}\right) \Delta \sim O(\tau)$, and therefore has a timedependent relationship associated with increased restriction with time. Using the propagator displacements due to diffusion, $O(10 \mu \mathrm{m})$, it is evident from fig. 1 that on this length scale there are multiple populations within the excitation slice and therefore a decreasing effective diffusion with time as is well known for porous media [37] is expected.

In the presence of flow a two-component model is used which accounts for the addition of a convective dispersion component

$$
\left\langle\boldsymbol{x}^{2}(\Delta)\right\rangle=2 D_{R} \Delta+K_{\alpha} \Delta^{\alpha}
$$

with a linear addition of a diffusive and ballistic motion ( $\alpha=2$ ) terms, where $K_{2}=\left\langle U^{2}\right\rangle[60]$ assumes an independence and separation of scales. Figure 12 shows eq. (10) and eq. (11) compared with the parallel-to-flow direction variance data for the homogeneous and heterogeneous gels at flow rates of $0 \mathrm{~mL} / \mathrm{h}$ and $30 \mathrm{~mL} / \mathrm{h}$, where $D_{R}$ is obtained from the average of the stationary flow dispersion data, $D_{\|} / D_{w}=0.86$, for the homogeneous gel and $\left\langle U^{2}\right\rangle=(106.1 \mu \mathrm{m} / \mathrm{s})^{2}$. To fit eq. (11) to the heterogeneous gel $\alpha=2$ fits well, while for the homogeneous gel $\alpha=1.86$ fits the data well. Both models use $K_{2}=\left\langle U^{2}\right\rangle$. These equations form an envelope for the data and it is therefore reasonable to assume that the normalized dispersion coefficients (fig. 11) fall within a description which accounts for a ballistic motion and restricted diffusion. The former causes an increasing dispersion coefficient with time while the latter causes a constant dispersion coefficient with time. This relationship accounts for the trending for the homogeneous data and to some extent explains the heterogeneous gel data, which after encountering the effects of an initial restriction, trends up due to the convective component. However at a long enough time $(\Delta>200 \mathrm{~ms})$ a second restriction affects the data, which corresponds to the effect seen for the propagators in fig. 9 and was discussed above using the two-population model. It is these restrictions on the time/length scale of solvent displacement which cause the non-trivial relationship between the dispersion and variance data. 


\section{Conclusions}

This study of $\beta$-lactoglobulin gels has used dynamic NMR experiments to probe the solvent dynamics through two different gel structures, heterogeneous $(p \mathrm{H}=5.2)$ and homogeneous $(p \mathrm{H}=7.0)$. The wealth of information that can be gleaned about the internal gel structure and solvent dynamics has been demonstrated, and the difficulties in analyzing a non-rigid porous medium which for the homogeneous gel experiences significant deformation and at high enough hydrodynamic stresses tears, has also been discussed. The solvent dynamics through the homogeneous gel showed Gaussian-like probability distributions of displacement on the length and time scales investigated, while the heterogeneous gel exhibited significant back-bone flow and at least two displacement/time dependent solvent structure restrictions seem present allowing the measurement of important gel structure length scales.

\section{References}

1. M. Annaka, T. Tanaka, Nature 355, 430 (1992).

2. F. Ilmain, T. Tanaka, E. Kokufuta, Nature 349, 400 (1991).

3. K.Y. Lee, D.J. Mooney, Chem. Rev. 101, 1869 (2001).

4. O. Okay, Prog. Polym. Sci. 25, 711 (2000).

5. Y. Qiu, K. Park, Adv. Drug Deliv. Rev. 53, 321 (2001).

6. N.M. Sangeetha, U. Maitra, Chem. Soc. Rev. 34, 821 (2005).

7. A.M. Stephan, Eur. Polym. J. 42, 21 (2006).

8. P.J. Flory, Faraday Discuss. Chem. Soc. 57, 7 (1974).

9. A.R. Altenberger, M. Tirrell, J. Chem. Phys. 80, 2208 (1984).

10. R.I. Cukier, Macromolecules 17, 252 (1984).

11. P.G. De Gennes, Macromolecules 19, 1245 (1986).

12. R.G. Bryant, Annu. Rev. Biophys. Biomol. Struct. 25, 29 (1996).

13. V. Klepko, Y. Melnichenko, V. Shilov, Polym. Gels Netw. 4, 351 (1996).

14. L. Masaro, X.X. Zhu, Prog. Polym. Sci. 24, 731 (1999).

15. P.I. Hurtado, L. Berthier, W. Kob, Phys. Rev. Lett. 98, 4 (2007).

16. Y.Y. Suzuki, M. Tokita, S. Mukai, Eur. Phys. J. E 29, 415 (2009).

17. P.G. De Gennes, Scaling Concepts in Polymer Physics (Cornell University Press, Ithaca, 1979).

18. M.A. Lauffer, Biophys. J. 1, 205 (1961).

19. P.G. De Gennes, J. Chem. Phys. 55, 572 (1971).

20. K.F. Arndt, D. Kuckling, A. Richter, Polym. Adv. Tech. 11, 496 (2000).

21. A. Richter et al., Sensors 8, 561 (2008).

22. S.H. Gehrke, Adv. Polym. Sci. 110, 81 (1993).
23. L. Sawyer, G. Kontopidis, Biochim. Biophys. Acta-Protein Struct. Molec. Enzym. 1482, 136 (2000).

24. J. Visser, T.J.M. Jeurnink, Exp. Therm. Fluid Sci. 14, 407 (1997).

25. K. Baussay et al., Int. J. Bio. Macromol. 34, 21 (2004).

26. C. Le Bon, D. Durand, T. Nicolai, Int. Dairy J. 12, 671 (2002).

27. T. Nicolai, D. Durand, Curr. Opin. Colloid Interface Sci. 12, 23 (2007).

28. S. Mehalebi, T. Nicolai, D. Durand, Int. J. Bio. Macromol. 43, 129 (2008).

29. A.H. Clark, G.M. Kavanagh, S.B. Ross-Murphy, Food Hydrocolloids 15, 383 (2001).

30. M. Langton, A.M. Hermansson, Food Hydrocolloids 5, 523 (1992).

31. P. Aymard et al., J. Chem. Phys. 93, 987 (1996).

32. C. Le Bon, T. Nicolai, D. Durand, Macromolecules 32, 6120 (1999).

33. P.T. Callaghan, Principles of Nuclear Magnetic Resonance Microscopy (Oxford University Press, New York, 1991).

34. A. Suzuki, M. Yoshikawa, J. Chem. Phys. 125 (2006).

35. N. Loren, M. Nyden, A.-M. Hermansson, Adv. Colloid Interface Sci. 150, 5 (2009).

36. N. Loren et al., Biomacromol. 10, 275 (2009).

37. P.N. Sen, Concepts Magn. Reson. Part A 23A, 1 (2004).

38. P.P. Mitra, P.N. Sen, L.M. Schwartz, Phys. Rev. B 47, 8565 (1993).

39. P.G. Saffman, Stud. Appl. Math. 50, 93 (1971).

40. G.I. Taylor, Proc. R. Soc. London, Ser. A 219, 186 (1953).

41. J. Bear, Dynamics of Fluids in Porous Media (Dover, New York, 1972) p. 764.

42. P.G. Saffman, J. Fluid Mech. 6, 321 (1959).

43. R.P. Chhabra, J. Comiti, I. Machac, Chem. Eng. Sci. 56, 1 (2001).

44. J.D. Seymour, P.T. Callaghan, AICHE J. 43, 2096 (1997).

45. R. Metzler, J. Klafter, Phys. Rep. 339, 1 (2000).

46. J.H. Cushman, T.R. Ginn, Water Resour. Res. 36, 3763 (2000).

47. D.L. Koch, J.F. Brady, Phys. Fluids 31, 965 (1988).

48. B. Berkowitz, Adv. Water Resour. 25, 861 (2002).

49. B. Berkowitz et al., Water Resour. Res. 38 (2002).

50. D.L. Koch, J.F. Brady, J. Fluid Mech. 180, 387 (1987).

51. B. Berkowitz et al., Rev. Geophys. 44, 49 (2006).

52. A. Compte, Phys. Rev. E 55, 6821 (1997).

53. R. Metzler, A. Compte, J. Phys. Chem. B 104, 3858 (2000).

54. J.D. Seymour et al., Phys. Rev. Lett. 93, 4 (2004).

55. M. Kopf et al., Biophys. J. 70, 2950 (1996).

56. E.O. Stejskal, J.E. Tanner, J. Chem. Phys. 42, 288 (1965).

57. B. Blumich, NMR Imaging of Materials (Clarendon Press, Oxford, 2000).

58. M. Sahimi, Applications of Percolation Theory (Taylor \& Francis, Bristol, 1994).

59. D. Kandhai et al., Phys. Rev. Lett. 88, 4 (2002).

60. J.P. Boon, S. Yip, Molecular Hydrodynamics (Dover, New York, 1980). 